\title{
Fabrication of Self-Supporting Annular Apertures for Use in the Transmission Electron Microscope
}

\author{
Liya $\mathrm{Yu}^{1}$, Aaron C. Johnston-Peck ${ }^{2}$, Andrew A. Herzing ${ }^{2}$ and Vincent Luciani ${ }^{1}$ \\ 1. Center for Nanoscale Science and Technology, National Institute of Standards Technology, \\ Gaithersburg, MD 20899 USA \\ 2. Material Measurement Laboratory, National Institute of Standards and Technology, Gaithersburg, \\ MD, 20899, USA
}

Apertures provide a way to further tailor the action of the transmission electron microscope (TEM). Recently there have been many reports looking at custom designed apertures, this was spurred by the discovery that an electron beam with quantized orbital angular momentum could be generated [1]. While much of the recent aperture design and fabrication work has been geared towards this endeavor many other applications for custom apertures exist. For instance, annular apertures can act as filters in the objective lens back focal plane to generate dark field images [2]. A focus ion beam (FIB) is typically used for fabrication of annular apertures due to the simplicity of processing. However, this approach limits the complexity of designs and requires three support arms in addition to the central beam stop, as shown in Figure 1(a). These unwanted extensions of the diaphragm eliminate electron transmission and can create unwanted diffraction effects, a particularly important consideration if the aperture is to function as the probe-forming aperture in the scanning TEM. Therefore, it would be beneficial to eliminate the need for support arms from the aperture design.

A self-supporting annular aperture has been proposed, as illustrated in Figure 1(b), and fabricated in different dimensions to validate simulation results (Figure 2). Rather than thick periodically spaced metal arm, the central beam stop is supported by a thin layer of amorphous SiN. The SiN provides the necessary mechanical support but interferes little with the transmitted electrons. Apart from the FIB approach, i.e., directly ion-milling the design into bulk metal, fabrication techniques which allow layer by layer building of structures, are introduced to produce this free standing center block without the inclusion of electron absorbing supports. LPCVD nitride was deposited, patterned and etched to form the membrane window acting as the supporting structure. Photolithography provides an effective and flexible means of patterning, which even practically enables the fabrication of diaphragms with multiple aperture designs. Gold was selected to be the electron absorbing material for its high atomic number and low stacking strain behavior. Instead of a plasma or ion-milling etch, wet etch chemistry was chosen as a low energy noble metal etch method in order to preserve the integrity of the thin SiN window.

The annular apertures fabricated by FIB and lithography were installed in a transmission electron microscope and their performance was compared through a series of tests. Additionally, their performance was modeled. Figure 2(d) and (e) show the calculation of point spread functions for two different designs. Degree of interaction between $\mathrm{SiN}$ and electrons which has implications for performance will also be evaluated.

\section{References:}

[1] B.J. McMorran et al, Science 331 (2011), p. 192.

[2] S. Bals, R. Kilass and C. Kisielowski, Ultramicroscopy 104 (2005), p. 281. 
[3] A.C.J.-P. acknowledges support of the National Research Council Postdoctoral Research Associateship Program.

(a)

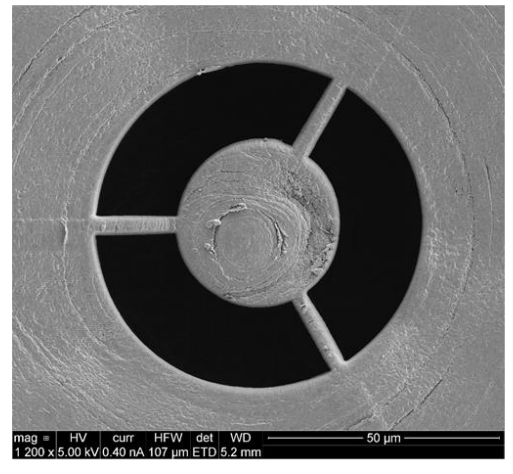

(b)

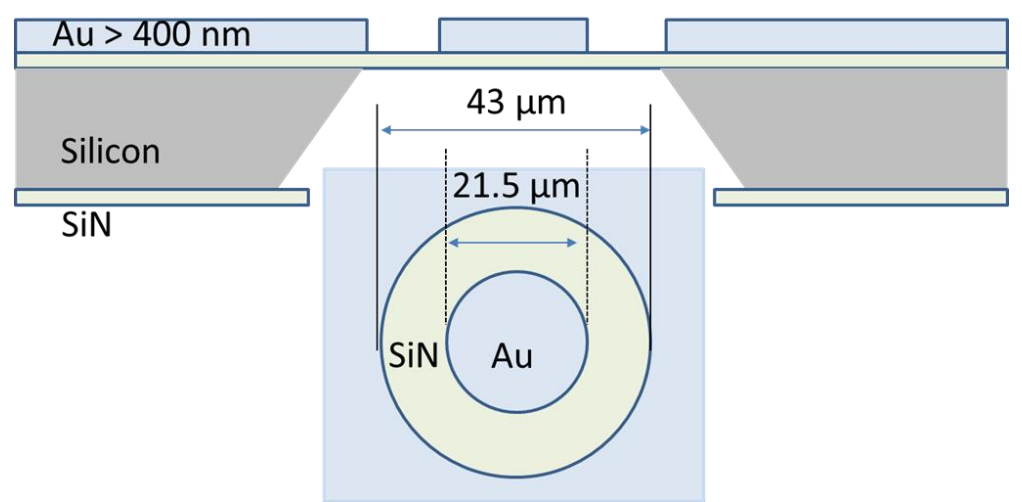

Figure 1 (a) SEM image of an annular aperture fabricated using FIB. (b) Newly proposed structure of self-support annular aperture.

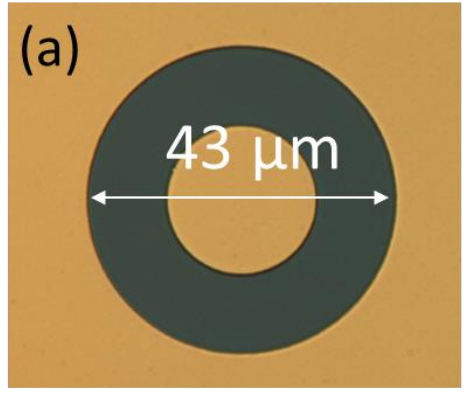

(c)

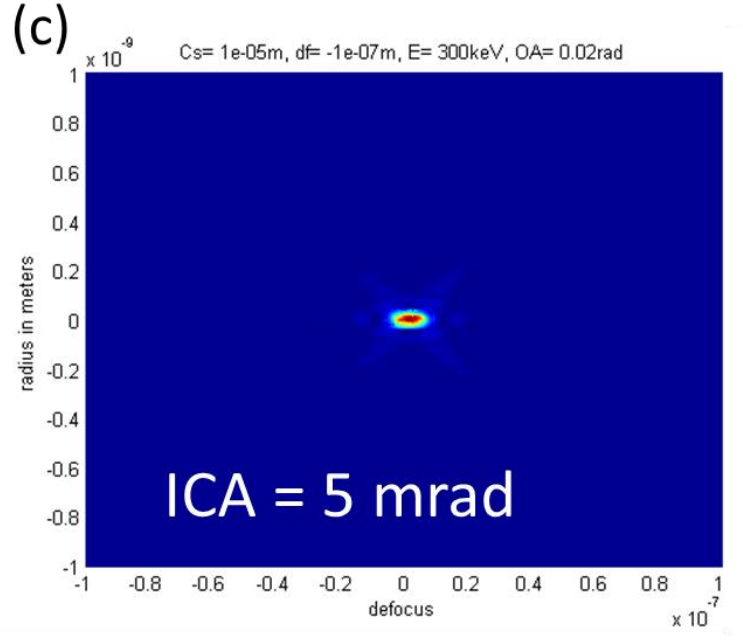

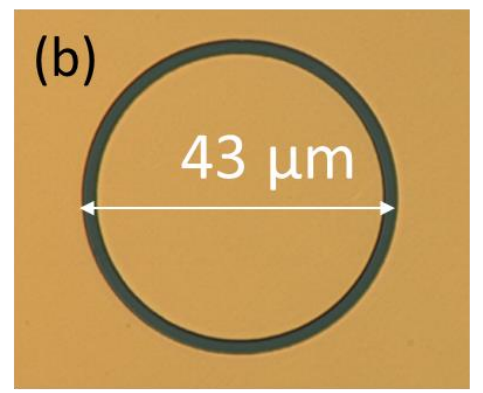

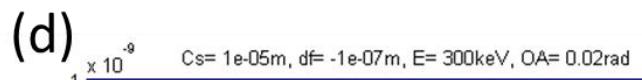

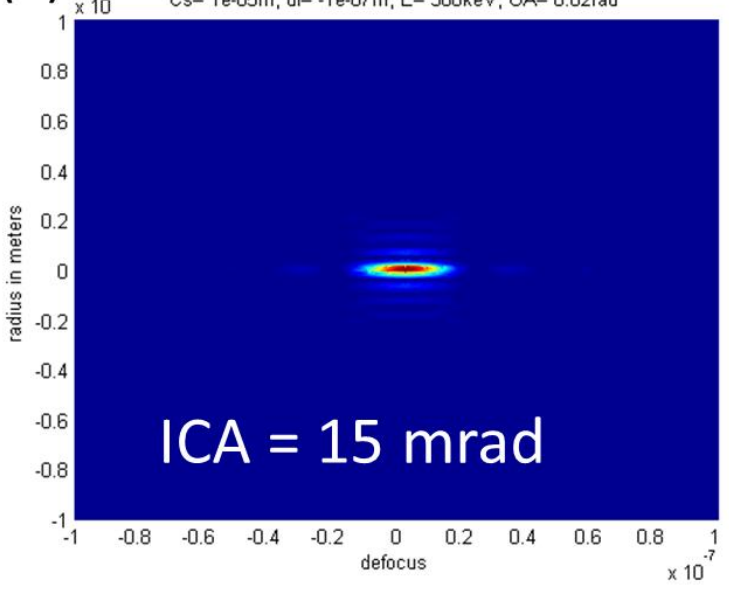

Figure 2 (a)-(b) New style annular aperture fabricated with different inner diameters. (c)-(d) Simulated point spread function as a function of inner convergence angle (ICA). 